\title{
Long-term neuropsychological outcome following pediatric anti-NMDAR encephalitis
}

Marienke A.A.M. de Bruijn, MD, Femke K. Aarsen, PhD, Marielle P. van Oosterhout, Marieke M. van der Knoop, MSc, Coriene E. Catsman-Berrevoets, MD, PhD, Marco W.J. Schreurs, PhD, Danielle E.M. Bastiaansen, MD, Peter A.E. Sillevis Smitt, MD, PhD, Rinze F. Neuteboom, MD, PhD, and Maarten J. Titulaer, MD, PhD, On behalf of the CHANCE Study Group

Neurology ${ }^{\circledR}$ 2018;90:e1997-e2005. doi:10.1212/WNL.0000000000005605

\section{Abstract}

\section{Objective}

To provide detailed long-term outcome data of children and adolescents following pediatric anti$N$-methyl-D-aspartate receptor (anti-NMDAR) encephalitis, to identify neuropsychological impairments, and to evaluate the influence of these factors on quality of life ( $\mathrm{oL}$ ).

\section{Methods}

All Dutch children diagnosed with anti-NMDAR encephalitis were identified. Patients currently aged 4 years or older were included in the follow-up study, consisting of a visit to our clinic for a detailed interview and a standardized neuropsychological assessment. The following domains were included: attention, memory, language, executive functioning, QoL, and fatigue. Primary outcome measures were $z$ scores on sustained attention, long-term verbal memory, QoL, fatigue, and working memory.

\section{Results}

Twenty-eight patients were included. Median Pediatric Cerebral Performance Category at last visit was 1 (interquartile range $1-2$, range $1-4)$, and $64 \%(18 / 28)$ of patients returned consistently to their previous school level. Twenty-two patients were included in the cross-sectional part of the long-term follow-up study. Median follow-up time was 31 months (interquartile range 15-49, range 5-91). There were problems with sustained attention $(z=-2.10,95 \%$ confidence interval $=-2.71$ to $-1.46, p<0.0001)$ and fatigue $(z=-0.96,95 \%$ confidence interval $=-1.64$ to $-0.28, p=0.008)$. Cognitive deficits were not correlated with QoL, while fatigue was strongly correlated with $\mathrm{QoL}(r=0.82, p<0.0001)$.

\section{Conclusions}

Although follow-up is often reported as "good" following pediatric anti-NMDAR encephalitis, many patients have cognitive problems and fatigue, even up until adolescence, resulting in academic achievement problems and lower QoL. For physicians, it is essential to be aware of these problems, to provide valuable advice to patients and caregivers in the acute and follow-up phase, and to consider early neuropsychological counseling.

\author{
Correspondence \\ Dr. Titulaer \\ m.titulaer@erasmusmc.nl
}

ค Podcast

Dr. David Lapides talks with Dr. Maarten Titulaer about his paper on neuropsychological outcome following pediatric anti-NMDAR encephalitis.

NPub.org/enhbn2

\section{- CME Course}

NPub.org/cmelist 


\section{Glossary}

anti-NMDAR = anti- $\mathrm{N}$-methyl-d-aspartate receptor; BRIEF = Behavior Rating Inventory of Executive Function; CANTAB = Cambridge Neuropsychological Test Automated Battery; HSV = herpes simplex virus; ICU = intensive care unit; IQR = interquartile range; mRS = modified Rankin Scale; PedsQL = Pediatric Quality of Life; PedsQL-MFS = Pediatric Quality of Life Multidimensional Fatigue Scale; PCPC = Pediatric Cerebral Performance Category; QoL = quality of life; RAVLT = Rey Auditory Verbal Learning Test.

Anti- $N$-methyl-D-aspartate receptor (anti-NMDAR) encephalitis is an autoimmune disorder, initially described in $2007 .^{1}$ Increased awareness has led to more frequent diagnoses, and currently more than 1,000 patients have been reported, of whom $35 \%$ are children. ${ }^{2}$ The disease course can be severe, with intensive care unit (ICU) admission in $75 \%$ of children. Nevertheless, if treated with adequate immunotherapy, outcome is considered favorable in $85 \%$ of children. ${ }^{2}$

However, there are signals that actual recovery might be less positive than initially reported. Small studies in both adults and children describe substantial deficits in multiple cognitive domains and also behavioral problems. ${ }^{3-9}$ Given these findings, it seems that despite apparent good outcome, full neuropsychological recovery is certainly not always achieved.

Functioning can be studied from different perspectives, ${ }^{10}$ including activities and participation. Outcome of anti-NMDAR encephalitis is currently measured in terms of activities with relatively crude measures, such as the modified Rankin Scale (mRS), ${ }^{11}$ while participation and quality of life ( $\left.\mathrm{QLL}\right)$ are also of major importance, especially in children and adolescents. Neuropsychological deficits can seriously affect participation and career choices as transition into adulthood might call for full cognitive abilities.

Therefore, the aim of this nationwide Dutch cohort study was to provide more insight into long-term outcome following pediatric anti-NMDAR encephalitis, with special emphasis on neuropsychological outcome, and to evaluate whether these neuropsychological factors influence QoL.

\section{Methods}

\section{Patients}

The Departments of Neurology and Pediatric Neurology of the Erasmus University Medical Center-Sophia Children's Hospital, Rotterdam, the Netherlands, are national referral sites for patients with suspected autoimmune encephalitis. In addition, the Department of Immunology is the national referral site for antineuronal antibody testing of samples from patients with suspected autoimmune encephalitis. Therefore, we had the opportunity to identify all Dutch children diagnosed with anti-NMDAR encephalitis, from January 2008 until March 2017, aged 0 to 18 years at disease onset. NMDAR antibodies were confirmed in serum and/or CSF by both commercial cell-based assay and immunohistochemistry.

\section{Clinical information}

Data about disease course were obtained from medical records and from detailed interviews with patients and caregivers during a visit to our clinic. Neurologic level of function was determined using the Pediatric Cerebral Performance Category (PCPC) scale (table e-1, links.lww.com/WNL/ A495). ${ }^{12}$

\section{Standard protocol approvals, registrations, and patient consents}

The institutional review board of the Erasmus University Medical Center approved the study protocol. Informed consent was obtained from adult patients and for children from their parents, and if applicable, also from children aged 12 to 18 years.

\section{Cross-sectional follow-up study}

All patients currently aged 4 years or older were approached to participate in the follow-up study, as neuropsychological testing and the questionnaires required a minimal age for participation. Patients were invited for a visit to our clinic, in which current complaints and level of functioning were discussed. In addition, patients underwent a standardized neuropsychological assessment. If a visit was not possible, current problems were discussed by phone, and questionnaires were sent to us by mail and checked in additional calls if necessary.

\section{Neuropsychological assessment}

The neuropsychological assessment consisted of a selection of the Cambridge Neuropsychological Test Automated Battery (CANTAB Research Suite 6.0, Cambridge Cognition Ltd., Cambridge, UK), additional neuropsychological tests, and questionnaires (table e-2, links.lww.com/WNL/A495). Tests and questionnaires were selected based on our own experiences and on disorders found in prior studies, were administrated in their Dutch versions, and are reliable and validated in the Netherlands. The tests and questionnaires were administered to assess skills in 6 domains:

1. Attention: Reaction Time (CANTAB), Dutch Dot Cancellation Test (Bourdon-Vos). ${ }^{13}$

2. Memory: Paired Associated Learning (CANTAB), Rey Auditory Verbal Learning Test (RAVLT). ${ }^{14}$

3. Language: Boston Naming Test, ${ }^{15}$ Token Test. ${ }^{16}$

4. Executive functioning: Intra-Extra Dimensional Set Shift, Spatial Span, Stockings of Cambridge (all CANTAB), Word Generation (NEPSY-II [ A Developmental Neuropsychological Assessment, Second Edition]), ${ }^{17}$ Behavior 
Rating Inventory of Executive Function (BRIEF-SelfReport and BRIEF-Adult) questionnaire, ${ }^{18}$ Strength and Difficulties Questionnaire (self-report and parent-proxy report). ${ }^{19}$

5. QoL: Pediatric Quality of Life Inventory 4.0 (PedsQL Self-Report and PedsQL Parent Proxy-Report). ${ }^{20}$

6. Fatigue: PedsQL Multidimensional Fatigue Scale questionnaire (PedsQL-MFS Self-Report and PedsQL-MFS Parent Proxy-Report). ${ }^{21}$

\section{Statistical analysis}

For group comparisons, we used the Mann-Whitney $U$ test (age), Fisher exact test (sex, immunotherapy), FisherFreeman-Halton extension (PCPC), and the Kruskal-Wallis one-way analysis of variance (character profiles). Results of neuropsychological assessments were compared with normative data of healthy individuals, corrected for age, sex, and educational level. Normative data for the CANTAB were obtained by CANTAB, Cambridge, UK. Scores were converted into standardized $z$ scores for comparison. For statistics, $z$ scores were set on minimum of -3 and maximum of +3 to prevent statistical differences by outliers (winsorization). In the graphs, the uncorrected $z$ scores are shown, but corrected $z$ scores were used for statistics. Displayed correlations were also calculated with corrected $z$ scores. The $z$ scores were analyzed using a one-sample $t$ test (test value $=0$ ). Primary outcome measures were sustained attention (Dutch Dot Cancellation Test-attention fluctuations), long-term verbal memory (RAVLT-Delayed Recall), fatigue (PedsQL-MFS Self-Report-Total Score), QoL (PedsQL Self-Report-Total Score), and working memory (BRIEF-Self-Report-Working Memory). Primary outcome measures were considered significant if $p<0.017$ (Bonferroni). For the secondary outcome measures of the neuropsychological assessment, $p$ values $<0.005$ were considered significant. Values between 0.005 and 0.05 should be interpreted carefully and considered exploratory. The relationship between our primary outcome measures and QoL were computed with a two-sided Pearson correlation coefficient. SPSS version 21.0 (IBM Corp., Armonk, NY) was used for statistical analyses, as well as GraphPad Prism 7 (GraphPad Software, La Jolla, CA) for Windows.

\section{Data availability}

Any data not published within this article are available at Erasmus University Medical Center. Patient-related data will be shared on request from any qualified investigator, maintaining anonymization of the individual patients.

\section{Results}

\section{Clinical characteristics}

Thirty children were identified, of whom 28 were included (for patient selection, see figure 1). Twenty-one patients were female $(75 \%)$, mainly in those aged 12 years or older $(89 \%)$. Median age at onset was 14 years. Eighteen patients (64\%)

\section{Figure 1 Flowchart of patient selection}

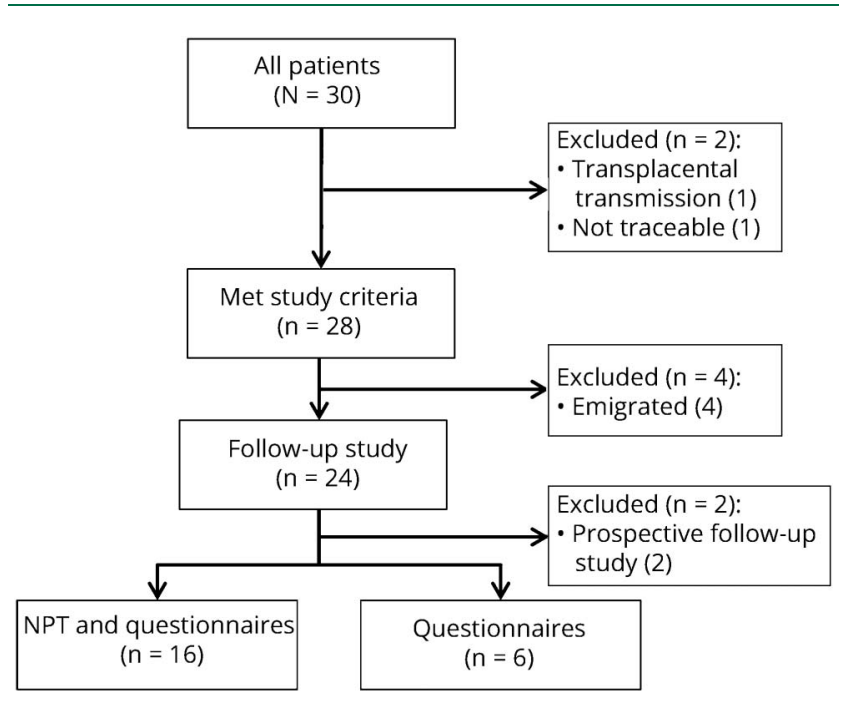

One patient was excluded because he was younger than 4 years (transplacental transmission of anti- $N$-methyl-D-aspartate receptor), ${ }^{31}$ and one patient was untraceable. Twenty-four patients participated in the follow-up study, of whom 2 are followed prospectively. Sixteen of the 22 participants completed the full neuropsychological assessment, 6 patients only completed the questionnaires, 3 visited our clinic, and 3 were contacted by phone because of geographical distance. NPT = neuropsychological testing.

reported a prodromal phase, including headache, blurred vision, or upper respiratory infection. Three children (11\%) developed anti-NMDAR encephalitis 3 to 7 weeks after a herpes simplex virus (HSV) type 1 encephalitis. In addition to those 3, one patient had a preexistent mild psychomotor developmental delay. The others were healthy before disease onset.

Most children presented with behavioral disorders (36\%) or seizures (36\%), less frequently with speech disorders and movement disorders. In 2 of 28 patients (7\%), hemiparesis was the presenting symptom, only occurring in children younger than 12 years (figure $2 \mathrm{~A}$ ). All patients presented to the initial physician with a maximum of 3 symptoms, while at maximum disease severity, 21 patients had developed more than 4 symptoms (figure 2, B and C). The numbers of symptoms between treatment and diagnosis were often comparable. Four patients developed one additional symptom after start of treatment, i.e., hypoventilation $(n=3)$ and bradycardia $(n=1)$. One patient developed seizures after diagnosis but before treatment, with a delay between diagnosis and treatment of 2 days (patient 16). One patient developed seizures 3 days after diagnosis and 9 days after initiation of treatment (patient 9).

Median time from symptom onset to maximum PCPC (maximum disease severity) was 30 days. Forty-six percent of patients $(13 / 28)$ were treated in the ICU with a median stay of 13 days. Total hospital stay was more than a month in $78 \%$ of patients. All patients were treated with first-line immunotherapy. Forty-six percent of patients received either 
A

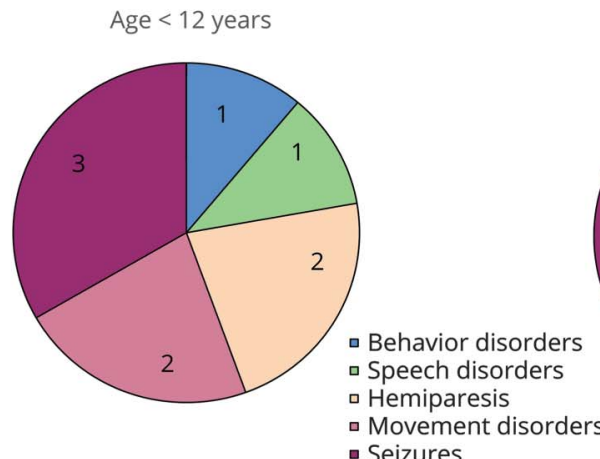

B

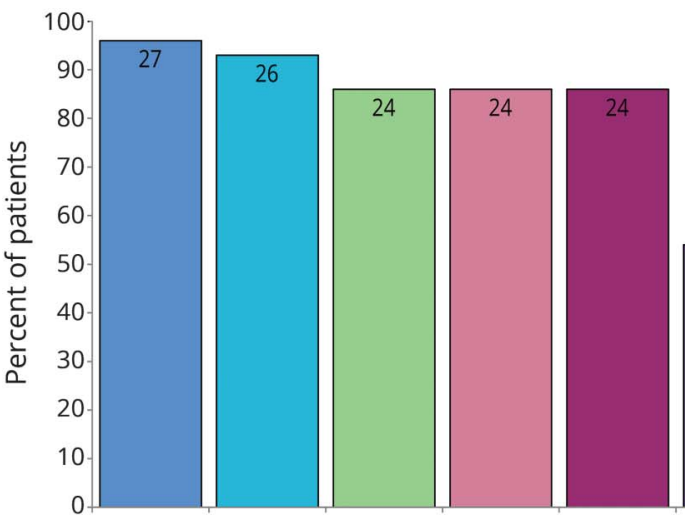

Age 12-18 years

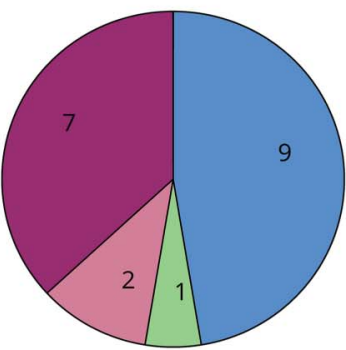

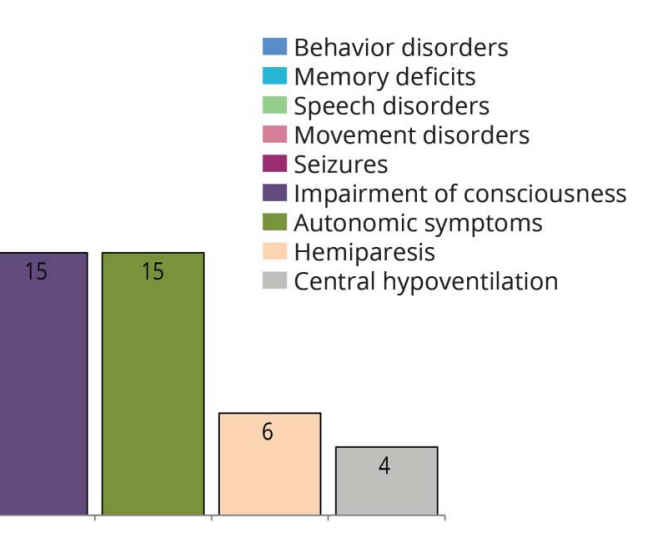

$c$

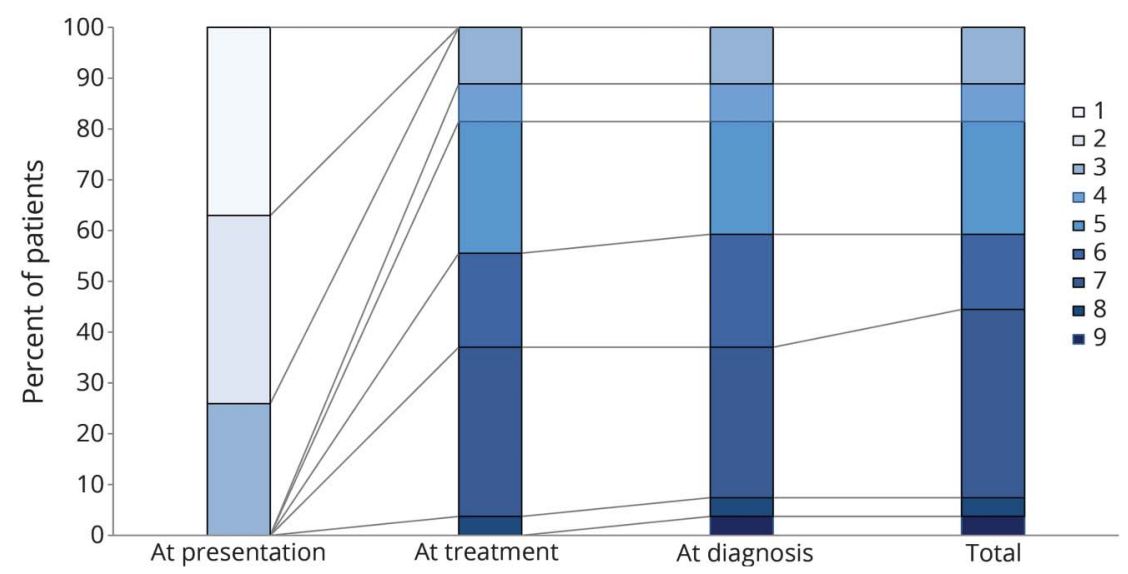

(A) Distribution of presenting symptoms of patients younger than 12 years and of patients aged 12-18years. (B) Cumulative symptoms during disease course. (C) Number of core symptoms at presentation, treatment, and antibody diagnosis, and total number of symptoms.

rituximab $(n=12)$ or cyclophosphamide $(n=1)$. In 14 of 28 patients (50\%), treatment was started before diagnosis, in 6 of 28 patients $(21 \%)$, treatment was initiated on the day of diagnosis, and in 8 of 28 patients (29\%), treatment was started after diagnosis. For all clinical characteristics, see table 1 and supplemental material (links.lww.com/WNL/A493).

\section{Outcome}

Three patients had a relapse 3,5 , and 35 months after first symptoms. One patient had a higher PCPC during the relapse than during the initial disease episode, leading to the initiation of rituximab. At hospital discharge, the median PCPC was 3 (interquartile range $[\mathrm{IQR}] 2-3$, range $1-4)$. Seventeen patients were discharged home, although 10 concurrently started with an outpatient rehabilitation program. Eleven patients (39\%) were transferred directly to an inpatient rehabilitation center. Median rehabilitation time was 98 days (IQR 58-194, range 34-578). The median PCPC at last visit was 1 (IQR 1-2, range 1-4). Twenty-six patients (93\%) resumed school after admission or rehabilitation. In 6 of the 26 patients $(23 \%)$ who resumed school, the current educational level was lower, including 5 patients with special educational needs. During follow-up, 3 patients stopped school prematurely because of fatigue $(n=2)$ or anxiety $(n=1)$. Overall, 
Table 1 Patient characteristics

\begin{tabular}{|c|c|}
\hline Sex, female & $21 / 28(75)$ \\
\hline Age $<12$ y & $4 / 9(44)$ \\
\hline Age $\geq 12$ y & $17 / 19(89)$ \\
\hline Age at onset, y & $14(7-17 ; 1-17)$ \\
\hline Prodromal phase & $18 / 28(64)$ \\
\hline Days to start of treatment & $21(9-65 ; 3-510)$ \\
\hline Days to antibody diagnosis & $27(13-61 ; 13-184)$ \\
\hline Days to maximum disease severity & $30(15-43 ; 2-94)$ \\
\hline \multicolumn{2}{|l|}{ Maximum PCPC } \\
\hline 3: Moderate disability & $1 / 28(4)$ \\
\hline 4: Severe disability & $16 / 28(57)$ \\
\hline 5: Coma/vegetative state & $11 / 28(39)$ \\
\hline ICU stay, d & $13(4-34 ; 1-45)$ \\
\hline Hospital stay, d & $55(33-67 ; 3-141)$ \\
\hline MRI abnormal ${ }^{a}$ & $10 / 27(37)$ \\
\hline CSF abnormal ${ }^{\mathrm{a}}$ & $21 / 27(78)$ \\
\hline EEG abnormal at presentation ${ }^{a}$ & $26 / 27(96)$ \\
\hline Ovarian teratoma suspected & $4 / 21(19)^{b}$ \\
\hline First-line IT & $28 / 28(100)$ \\
\hline Methylprednisolone & $27 / 28(96)$ \\
\hline Plasmapheresis & $6 / 28(21)$ \\
\hline Immunoglobulins & $21 / 28(75)$ \\
\hline Interval between first- and second-line IT, d & $18(14-41 ; 6-200)$ \\
\hline Second-line IT & $13 / 28(46)$ \\
\hline Rituximab & $12 / 28(43)$ \\
\hline Cyclophosphamide & $1 / 28(4)$ \\
\hline Cell-based assay anti-NMDAR serum ${ }^{a, c}$ & $16 / 24(67)$ \\
\hline Cell-based assay anti-NMDAR, CSF ${ }^{\mathrm{a}}$ & $27 / 27(100)$ \\
\hline
\end{tabular}

Abbreviations: anti-NMDAR = anti- $N$-methyl-D-aspartate receptor; ICU = intensive care unit; IT = immunotherapy; $\mathrm{PCPC}=$ pediatric cerebral performance category.

Data are $\mathrm{n} / \mathrm{n}(\%)$ or median (interquartile range; range).

additional details are shown online.

${ }^{b}$ For girls $\geq 12$ years: 4/19 (21\%). All 4 girls underwent resection; 3 had a teratoma, one a follicle cyst.

'In one patient, only serum was available; cell-based assay, immunohistochemistry, and live neurons were all positive.

18 of 28 patients (64\%) returned consistently to their previous school level.

\section{Cross-sectional follow-up study}

Twenty-two patients participated in the follow-up study, with a median follow-up time after symptom onset of 31 months (IQR 15-49, range 5-91). Nineteen were seen at our clinic, while 3 had an interview by phone. All 22 patients completed questionnaires, while 16 patients completed the full neuropsychological assessment (figure 1). Individual information is shown online in table e-3 (links.lww.com/WNL/A495). Median age at last visit was 17 years (IQR 12-19, range 4-25). Three patients had post-HSV encephalitis anti-NMDAR encephalitis, 2 with a follow-up PCPC of 3 (patients 14 and 18) and 1 with a PCPC of 4 (patient 19). One patient had a PCPC of 4 because of spasticity and vocal cord paralysis (patient 6).

\section{Neuropsychological outcome}

Characteristics of the 16 patients who underwent full neuropsychological assessment were similar to those of the other patients ( $\mathrm{n}=13$; table e-4, links.lww.com/WNL/A495). Patients had lower sustained attention scores $(z=-2.10$, $p_{\text {uncorrected }}<0.0001$; table 2 ), and these were consistent among almost all patients. The mean score on long-term verbal memory tended to be lower $\left(z=-0.68, p_{\text {uncorrected }}=\right.$ $0.031)$. Patients reported more fatigue $\left(z=-0.96, p_{\text {uncorrected }}\right.$ $=0.008)$, and QoL tended to be lower $\left(z=-0.87, p_{\text {uncorrected }}=\right.$ $0.032)$, while working memory was not different $(z=0.24$, $\left.p_{\text {uncorrected }}=0.23\right)$. Results were similar when the 3 patients with anti-NMDAR encephalitis post HSV encephalitis were excluded (1 full neuropsychological assessment, 2 only completed questionnaires; data not shown).

There was a strong correlation between self-reported fatigue and QoL ( $r=0.82, p<0.0001$; figure 3), also as reported by parents (Parent Proxy-Report-Total Score; $r=0.70, p=$ 0.004). There were no significant correlations between $\mathrm{QoL}$ and fatigue and the cognitive domains sustained attention and long-term verbal memory (figure 3 ). Treatment delay, followup time, age at onset, ICU stay, maximum PCPC, and PCPC at follow-up were not correlated with sustained attention, long-term verbal memory, or fatigue (figure e-1, links.lww. com/WNL/A494). Sustained attention and long-term verbal memory were also not correlated with QoL scores as reported by parents (sustained attention: $r=0.20, p=0.62$; long-term verbal memory: $r=0.45, p=0.27$ ).

Among the secondary outcome measures (tables e-5 and e-6, links.lww.com/WNL/A495), the mean $z$ score on domain speed was lower (Dutch Dot Cancellation Test-reaction time; $z=-1.53$, $p_{\text {uncorrected }}=0.002$ ). Scores on the domains visual memory (Paired Associated Learning-total errors; $z=$ -0.90, $\left.p_{\text {uncorrected }}=0.016\right)$, short-term verbal memory (RAVLT Trials 1-5); $z=-0.76, p_{\text {uncorrected }}=0.023$ ), and naming (Boston Naming Test-total score; $z=-0.78$, $\left.p_{\text {uncorrected }}=0.019\right)$ were low, but between 0.05 and 0.005 .

Results of the questionnaires completed by parents were comparable to those of children (table e-7, links.lww.com/ WNL/A495).

Patients and parents mentioned similar difficulties in the detailed interview (17/22). Regarding school or work performance, the most notable problems were word finding difficulties (24\%), dyslexia (12\%), and attention and concentration deficits 
Table 2 Results of primary outcome measures

\begin{tabular}{|c|c|c|c|c|c|c|}
\hline Domain; test; measure & No. & $z$ Score, mean & $95 \% \mathrm{Cl}$ & $z$ Score $<0, \mathrm{n}(\%)$ & $z$ Score $<-2, \mathrm{n}(\%)$ & $p$ Value \\
\hline Sustained attention; DDCT; attention fluctuations & 16 & -2.10 & -2.71 to -1.48 & $15(94)$ & $10(63)$ & $<0.0001^{a}$ \\
\hline Long-term verbal memory; RAVLT-Delayed Recall & $15^{\mathrm{b}}$ & -0.68 & -1.29 to -0.07 & $12(80)$ & $3(20)$ & 0.031 \\
\hline Fatigue; PedsQL-MFS; Self-Report; Total Score & $21^{c}$ & -0.96 & -1.64 to -0.28 & $16(76)$ & $5(24)$ & $0.008^{\mathrm{a}}$ \\
\hline Quality of life; PedsQL; Self-Report; Total Score & $21^{c}$ & -0.86 & -1.64 to -0.08 & $15(71)$ & $7(33)$ & 0.032 \\
\hline Working memory; BRIEF; Working Memory & $19^{c}$ & 0.24 & -0.17 to 0.65 & $8(42)$ & 0 & 0.23 \\
\hline
\end{tabular}

Abbreviations: BRIEF = Behavior Rating Inventory of Executive Function; $\mathrm{Cl}=$ confidence interval; $\mathrm{DDCT}=$ Dutch Dot Cancellation Test; $\mathrm{MFS}=$ Multidimensional Fatigue Scale; PedsQL = Pediatric Quality of Life; RAVLT = Rey Auditory Verbal Learning Test.

Exclusion of patient 6 (with vocal cord paralysis), patients 14,18 , and 19 (all post-herpes simplex virus encephalitis anti- $N$-methyl-D-aspartate receptor encephalitis), and patient 16 (with prior mild developmental delay) did not affect results.

${ }^{a} p<0.017$ (Bonferroni).

${ }^{\mathrm{b}}$ Of one patient (no. 6), no data are shown as the test was aborted because of vocal cord paralysis.

${ }^{\mathrm{c}}$ No normative data available for test results of the youngest patient(s).

(18\%). Other problems were impulsiveness (18\%), anxiety (18\%), and indecisiveness (12\%). Concerning the disease period, 21 of 22 patients (95\%) had a persistent (fragmented or complete) amnesia.

Based on our own observations during the visits to our clinics, we could differentiate 3 frontal lobe syndrome profiles using the character descriptions by parents and the main complaints of the patients themselves. This way, we allocated the patients visiting our clinic into 3 groups: (1) passive (apathy, $\mathrm{n}=5),(2)$ moderate (no signs of a frontal lobe syndrome, $\mathrm{n}=6$ ), and (3) active (impulsive, $\mathrm{n}=7$ ). The median scores on QoL and fatigue were compared between these groups (visualized in figure e-2, links.lww. com/WNL/A494). Among the passive patients, the school dropout rate was $80 \%(4 / 5)$, while for the active patients, school resumption was achieved in all 7, of whom 2 did not retain previous school level.

Figure 3 Overview of correlations between primary outcome measures
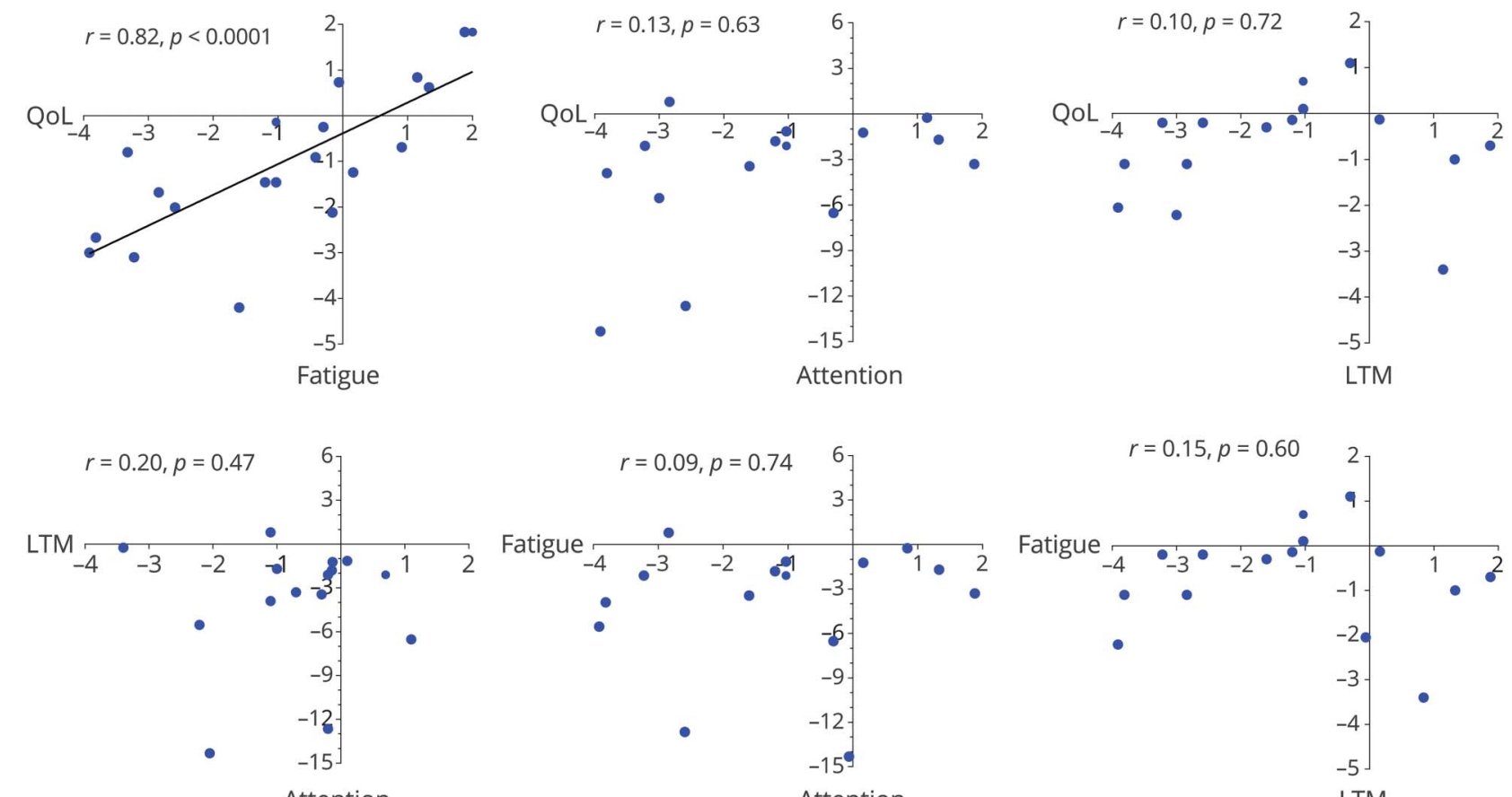

Attention

Attention

LTM

Outcome measures: sustained attention (Dutch Dot Cancellation Test-attention fluctuations), long-term verbal memory (RAVLT-Delayed Recall), fatigue (PedsQL-MFS Self-Report-Total Score), QoL (PedsQL Self-Report-Total Score), and working memory (BRIEF-Working Memory). In all graphs, results of uncorrected $z$ scores are shown, but the correlations are calculated with corrected $z$ scores (maximum 3, minimum -3 ). Anti-NMDAR = anti- $N$-methyl-Daspartate receptor; BRIEF = Behavior Rating Inventory of Executive Function; HSV = herpes simplex virus; LTM = long-term verbal memory; MFS = Multidimensional Fatigue Scale; PedsQL = Pediatric Quality of Life; QoL = quality of life; RAVLT = Rey Auditory Verbal Learning Test. 


\section{Discussion}

We have demonstrated that, despite good functional recovery (according to the mRS or PCPC), persistent cognitive deficits are common in young children and adolescents following pediatric anti-NMDAR encephalitis, and that important parameters for good outcome, such as treatment delay or age at onset, do not specifically affect neuropsychological outcome. Other interesting and important findings are that patients reported more fatigue, and that patients with fatigue also reported a poorer QoL, while poorer cognitive outcome did not affect QoL.

Fatigue has not been evaluated before in patients with antiNMDAR encephalitis. However, it is known to be a common disabling symptom in pediatric acquired brain injury, ${ }^{22,23}$ making our results that fatigue was associated with poorer QoL plausible. This finding is supported further by the frequent reporting of fatigue by patients as the most disabling symptom often hampering normal participation.

Remarkably, poorer cognitive outcome did not influence QoL, possibly because QoL questionnaires comprise general topics, while patients often reported specific task-related problems, which might be underestimated in current questionnaires. In addition, patients becoming accustomed to a new "stable" situation and reduced awareness might be other explanations. The latter is less likely because parents' QoL scores were comparable.

Predictors of good functional outcome such as treatment delay, maximum PCPC, and ICU stay were not correlated with QoL, fatigue, or sustained attention. This supports our statement that "good" outcome certainly not always means "good" total recovery. NMDAR antibodies are considered to compromise signal transmission, leading to problems in multiple functional networks, corresponding to the extent of symptoms. Finke et al. ${ }^{24}$ showed that a reduced connectivity of the anterior hippocampus and the anterior default mode network was associated with poorer memory in anti-NMDAR encephalitis. ${ }^{24}$ In addition, this reduced connectivity is also described in a broad spectrum of other neurologic conditions. ${ }^{25-28}$ These connections seem most vulnerable, which may explain the discrepancy between good outcome and poor memory recovery. A follow-up study testing patients by serial neuropsychological tests combined with fMRI will be essential to examine the correlation between cognitive functioning and this reduced connectivity over time, and to examine whether this process is reversible.

Most anti-NMDAR encephalitis follow-up studies concentrate on the neurobehavioral problems of disinhibition. However, frontal lobe syndromes are more widespread, and little is known about passive patients during rehabilitation and follow-up. Our data suggest that these "passive" patients might be more at risk to develop problems with normal participation because these patients showed more school dropout rates and reported more fatigue. This observation needs confirmation in future research, but may have important consequences for rehabilitation programs.

For cognitive outcome, we particularly observed lower scores in the domain sustained attention and speed. Possibly these cognitive deficits are most prominent and should be considered during cognitive rehabilitation. However, there was no correlation between the different cognitive test results, which underlines that the occurring cognitive deficits are diverse and probably different parts of the brain are affected. Short-term verbal memory and language scores were also lower. Apparently these domains are more vulnerable to dysfunction of the NMDAR. These findings are partially in concordance with earlier findings, ${ }^{3-9}$ although these previous published studies describe more diverse cognitive deficits, with additional deficits in executive functioning. However, these studies are difficult to interpret and to compare properly to our results because of limited patient numbers and unstandardized methods and because some patients were assessed in the acute disease phase. By using standardized performance-based measures, such as CANTAB, we found no prominent problems in executive functions. Nevertheless, by using rating measures (questionnaires, interviews), patients reported substantial difficulties in performing activities of daily living. An explanation for this disconnection is that performancebased measures and rating measures do not assess the same aspects in cognitive and behavioral functioning. Rating measures assess whether goals in activities of daily living are reached and have higher ecological validity. ${ }^{29}$ Next to the BRIEF (and other rating measures we performed), the BADSC (Behavioral Assessment of the Dysexecutive Syndrome in Children) might be a useful addition.

The present study, with national coverage, detailed description of clinical data, and the use of a systematic neuropsychological assessment, provides broad, valuable results, likely to be externally valid. This study exclusively pertains to pediatric anti-NMDAR encephalitis, also a valuable aspect, because in comparison to adults, there are differences in disease onset, treatment decisions, and social functioning. First, children present more often with seizures or behavioral changes, ${ }^{30}$ whereas adults mostly present with psychiatric symptoms or memory dysfunction, ${ }^{2}$ which may lead to different intervals to diagnosis and treatment. Second, treatment decisions can be age-dependent and may affect outcome; i.e., physicians tend to be more aggressive in children, starting immunotherapy early while simultaneously being more careful with cyclophosphamide. Third, neuropsychological problems can seriously affect participation as successful transition into adulthood calls for full cognitive, emotional, and behavioral abilities.

We had the unique opportunity to include all Dutch children with anti-NMDAR encephalitis. Nevertheless, despite national coverage and increasing incidence, anti-NMDAR encephalitis is a rare disease. Therefore, to include a sufficient 
number of patients with a reasonable follow-up time, a retrospective study design was inevitable but with the associated problems. The first issue is missing data. The amount of missing data was minimized by contacting treating physicians, parents, and patients. Regarding selection bias (between patients participating and nonparticipating in the follow-up study), we found no difference in clinical characteristics. Furthermore, clinical characteristics are in line with previous studies. $^{2,30}$ The results of the participants thus seem to be a good representation for the total group and results are probably generalizable.

Overall, our findings highlight the importance of awareness of persisting neuropsychological deficits and excessive fatigue following pediatric anti-NMDAR encephalitis. With a considerable median follow-up time (almost 3 years), our results clearly indicate that neuropsychological deficits can be prolonged. Currently, disease outcome is assessed with parameters measuring impairment and disabilities ( $\mathrm{mRS},{ }^{11}$ PCPC), and treatment decisions are based on these parameters. Our results show that neuropsychological parameters measuring participation and QoL are also important and should be considered when assessing outcome, because these factors can substantially affect participation and well-being. Therefore, physicians should inform patients and parents correctly about the occurrence of prolonged neuropsychological problems. In addition, they should provide good accessibility to neuropsychological counseling in rehabilitation centers immediately following the acute disease course and during follow-up.

\section{Author contributions}

M.A.A.M. de Bruijn: study design, acquisition of data, data analysis, medical writing. F.K. Aarsen: study design, acquisition of data, revising the manuscript for content. M.P. van Oosterhout: acquisition of data, revising the manuscript for content. M.M. van der Knoop: acquisition of data, revising the manuscript for content. C.E. Catsman-Berrevoets: revising the manuscript for content. M.W.J. Schreurs: revising the manuscript for content. D.E.M. Bastiaansen: acquisition of data, revising the manuscript for content. P.A.E. Sillevis Smitt: revising the manuscript for content. R.F. Neuteboom: study design, acquisition of data, revising the manuscript for content. M.J. Titulaer: study design, acquisition of data, data analysis, medical writing.

\section{Acknowledgment}

The authors gratefully thank all patients and their parents for their participation. They thank all referring physicians, with special thanks to Dr. F.M.C. Berkesteijn, Dr. J.M. de Bont, Prof. Dr. K.P.J. Braun, Prof. Dr. O.F. Brouwer, Dr. J.F.H.M. Claes, Dr. K.G.J. van Dijk, Dr. C. Erasmus, Dr. J.G.J. Hoeijmakers, Dr. W. Peper, Dr. J.P.A. Samijn, Dr. R.D. Thijs, Prof. Dr. R.J. Vermeulen. The authors thank Esther Hulsenboom and Mariska Nagtzaam for technical assistance.

\section{Study funding}

M.T. was supported by an Erasmus MC fellowship, has received funding from the Netherlands Organization for Scientific Research (NWO, Veni incentive), from the Dutch Epilepsy Foundation (NEF, project 14-19), and from ZonMw (Memorabel program).

\section{Disclosure}

M. de Bruijn, F. Aarsen, M. van Oosterhout, M. van der Knoop, C. Catsman-Berrevoets, M. Schreurs, and D. Bastiaansen report no disclosures relevant to the manuscript. P. Sillevis Smitt holds a patent for the detection of anti-DNER and received research support from EUROIMMUN. R. Neuteboom reports no disclosures relevant to the manuscript. M. Titulaer has filed a patent for methods for typing neurologic disorders and cancer, and devices for use therein, and has received research funds for serving on a scientific advisory board of MedImmune LLC, for consultation at Guidepoint Global LLC, and an unrestricted research grant from EUROIMMUN AG. Go to Neurology.org/N for full disclosures.

Received September 18, 2017. Accepted in final form March 15, 2018.

\section{References}

1. Dalmau J, Tuzun E, Wu HY, et al. Paraneoplastic anti-N-methyl-D-aspartate receptor encephalitis associated with ovarian teratoma. Ann Neurol 2007;61:25-36.

2. Titulaer MJ, McCracken L, Gabilondo I, et al. Treatment and prognostic factors for long-term outcome in patients with anti-NMDA receptor encephalitis: an observational cohort study. Lancet Neurol 2013;12:157-165.

3. Finke C, Kopp UA, Pruss H, Dalmau J, Wandinger KP, Ploner CJ. Cognitive deficits following anti-NMDA receptor encephalitis. J Neurol Neurosurg Psychiatry 2012;83: 195-198.

4. McKeon GL, Scott JG, Spooner DM, et al. Cognitive and social functioning deficits after anti-N-methyl-D-aspartate receptor encephalitis: an exploratory case series. J In Neuropsychol Soc 2016;22:828-838

5. Loughan AR, Allen A, Perna R, Malkin MG. Anti-N-methyl-D-aspartate receptor encephalitis: a review and neuropsychological case study. Clin Neuropsychol 2016, 30:150-163.

6. Hinkle CD, Porter JN, Waldron EJ, Klein H, Tranel D, Heffelfinger A. Neuropsychological characterization of three adolescent females with anti-NMDA receptor encephalitis in the acute, post-acute, and chronic phases: an inter-institutional case series. Clin Neuropsychol 2017;31:268-288.

7. Matricardi S, Patrini M, Freri E, et al. Cognitive and neuropsychological evolution in children with anti-NMDAR encephalitis. J Neurol 2016;263:765-771.

8. Iadisernia E, Battaglia FM, Vanadia E, Trapolino E, Vincent A, Biancheri R. Anti-Nmethyl-D-aspartate-receptor encephalitis: cognitive profile in two children. Eur J Paediatr Neurol 2012;16:79-82

9. McKeon GL, Robinson GA, Ryan AE, et al. Cognitive outcomes following anti-Nmethyl-D-aspartate receptor encephalitis: a systematic review. J Clin Exp Neuropsychol 2018;40:234-252.

10. World Health Organization. International Classification of Functioning, Disability and Health (ICF). Geneva: World Health Organization; 2001.

11. van Swieten JC, Koudstaal PJ, Visser MC, Schouten HJ, van Gijn J. Interobserver agreement for the assessment of handicap in stroke patients. Stroke 1988;19:604-607.

12. Fiser DH. Assessing the outcome of pediatric intensive care. J Pediatr 1992;121: $68-74$.

13. Vos PG. Bourdon-Vos Test Manual, 3rd ed. Amsterdam: Pearson; 1998.

14. van den Burg W, Kingma A. Performance of 225 Dutch school children on Rey's Auditory Verbal Learning Test (AVLT): parallel test-retest reliabilities with an in terval of 3 months and normative data. Arch Clin Neuropsychol 1999;14:545-559.

15. Storms G, Saerens J, De Deyn PP. Normative data for the Boston Naming Test in native Dutch-speaking Belgian children and the relation with intelligence. Brain Lang 2004;91:274-281.

16. Paquier PF, van Mourik M, van Dongen HR, Catsman-Berrevoets C, Creten WL, Van Borsel J. Normative data of 300 Dutch-speaking children on the Token Test. Aphasiology 2009;23:427-437.

17. Korkman MKU, Kemp S. Dutch Technical Manual of NEPSY II-NL. Amsterdam: Pearson; 2010.

18. Smidts DP, Huizinga M. Manual of BRIEF Executive Functions Behavior Questionnaire. Amsterdam: Hogrefe; 2009.

19. van Widenfelt BM, Goedhart AW, Treffers PD, Goodman R. Dutch version of the Strengths and Difficulties Questionnaire (SDQ). Eur Child Adolesc Psychiatry 2003; $12: 281-289$. 
20. Engelen V, Haentjens MM, Detmar SB, Koopman HM, Grootenhuis MA. Health related quality of life of Dutch children: psychometric properties of the PedsQL in the Netherlands. BMC Pediatr 2009;9:68.

21. Gordijn M, Cremers EM, Kaspers GJ, Gemke RJ. Fatigue in children: reliability and validity of the Dutch PedsQL Multidimensional Fatigue Scale. Qual Life Res 2011;20: 1103-1108.

22. Chevignard M, Francillette L, Toure H, et al. Academic outcome, participation and health-related quality of life following childhood severe traumatic brain injury: results of a prospective longitudinal study: the seven-year follow-up of the TGE cohort. Ann Phys Rehabil Med 2016;59S:e133.

23. Toussaint-Duyster LC, Wong YYM, Van der Cammen-van Zijp MH, et al. Fatigue and physical functioning in children with multiple sclerosis and acute disseminated encephalomyelitis. Mult Scler Epub 2017 April 1.

24. Finke C, Kopp UA, Scheel M, et al. Functional and structural brain changes in anti-N-methyl-D-aspartate receptor encephalitis. Ann Neurol 2013;74: 284-296.

25. Allen G, Barnard H, McColl R, et al. Reduced hippocampal functional connectivity in Alzheimer disease. Arch Neurol 2007;64:1482-1487.
26. Grydeland H, Walhovd KB, Westlye LT, et al. Amnesia following herpes simplex encephalitis: diffusion-tensor imaging uncovers reduced integrity of normal-appearing white matter. Radiology 2010;257:774-781.

27. Zhou Y, Liang M, Jiang T, et al. Functional dysconnectivity of the dorsolateral prefrontal cortex in first-episode schizophrenia using resting-state fMRI. Neurosci Lett 2007;417:297-302.

28. Shapira-Lichter I, Weinstein M, Lustgarten N, et al. Impaired diffusion tensor imaging findings in the corpus callosum and cingulum may underlie impaired learning and memory abilities in systemic lupus erythematosus. Lupus 2016;25:1200-1208.

29. Toplak ME, West RF, Stanovich KE. Practitioner review: do performance-based measures and ratings of executive function assess the same construct? J Child Psychol Psychiatry 2013;54:131-143.

30. Armangue T, Titulaer MJ, Malaga I, et al. Pediatric anti-N-methyl-D-aspartate receptor encephalitis: clinical analysis and novel findings in a series of 20 patients. J Pediatr 2013;162:850-856.e2.

31. Hilderink M, Titulaer MJ, Schreurs MW, Keizer K, Bunt JE. Transient anti-NMDAR encephalitis in a newborn infant due to transplacental transmission. Neurol Neuroimmunol Neuroinflamm 2015;2:e126. 


\title{
Long-term neuropsychological outcome following pediatric anti-NMDAR encephalitis
}

\author{
Marienke A.A.M. de Bruijn, MD, Femke K. Aarsen, PhD, Marielle P. van Oosterhout, \\ Marieke M. van der Knoop, MSc, Coriene E. Catsman-Berrevoets, MD, PhD, Marco W.J. Schreurs, PhD, \\ Danielle E.M. Bastiaansen, MD, Peter A.E. Sillevis Smitt, MD, PhD, Rinze F. Neuteboom, MD, PhD, \\ and Maarten J. Titulaer, MD, PhD, On behalf of the CHANCE Study Group
}

Correspondence Dr. Titulaer m.titulaer@erasmusmc.nl

Cite as: Neurology ${ }^{\circledR}$ 2018;90:e1997-e2005. doi:10.1212/WNL.0000000000005605

\section{Study question}

What are the long-term effects of pediatric anti-NMDAR encephalitis on neuropsychologic function and quality of life?

\section{Summary answer}

Many patients experienced cognitive problems and fatigue even after apparent recovery, resulting in decreased quality of life and impaired academic performance.

\section{What is known and what this paper adds}

Anti-NMDAR encephalitis is an autoimmune disorder, and the disease course is severe in $75 \%$ of pediatric cases. While previous studies have indicated good outcomes after immunotherapy, new evidence suggests that negative long-term effects may exist. The present study confirms these findings, and provides strong evidence demonstrating the long-term effects of pediatric antiNMDAR encephalitis. It suggests that academic performance is most affected by passivity as a sign of frontal lobe syndrome.

\section{Participants and setting}

Dutch children $(0-18$ years of age; $n=28)$ diagnosed with anti-NMDAR encephalitis between January 2008 and March 2017 were included in the initial study. Of these, 22 patients were included in the cross-sectional study.

\section{Design, size, and duration}

Clinical information was extracted from medical records and detailed clinical interviews. Outcome data were obtained during a cross-sectional follow-up phase in which participants completed an in-clinic standardized neuropsychologic assessment or a mailed questionnaire and telephone interview. Neuropsychologic results were converted into standardized z score and compared to normative data for healthy matched subjects.

\section{Primary outcomes}

Patient functioning was assessed using $\mathrm{z}$ scores in 5 domains: sustained attention, long-term verbal memory, working memory, quality of life, and fatigue.

\section{Main results and the role of chance}

The children were predominantly female $(75 \%)$ and $\geq 12$ years of age $(89 \%)$. The median age of onset was 14 years. Sixty-four
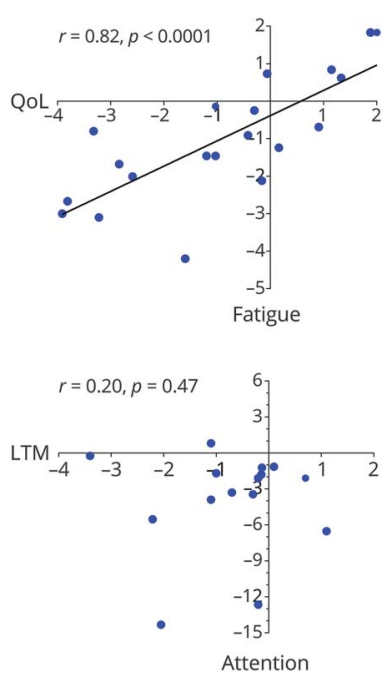

percent of patients returned to their previous school level. Sixteen children completed the neuropsychological assessment and showed impairments in sustained attention $(\mathrm{z}=-2.10$, $95 \% \mathrm{CI}=-2.71$ to $-1.46, p<0.0001)$ and fatigue $(\mathrm{z}=-0.96$, $95 \% \mathrm{CI}=-1.64$ to $-0.28, p=0.008)$. Fatigue, but not cognitive function, was strongly correlated with quality of life $(r=0.82$, $p<0.0001)$.

\section{Bias, confounding, and other reasons for caution}

The study included a small number of patients, especially in the cross-sectional phase.

\section{Generalizability to other populations}

The results can be generalized to other cases of pediatric antiNMDAR encephalitis.

\section{Study funding/potential competing interests}

The study was funded in part by the Netherlands Organization for Scientific Research, the Dutch Epilepsy Foundation, and ZonMW. Go to Neurology.org/N for full disclosures. 


\section{Neurology}

\section{Long-term neuropsychological outcome following pediatric anti-NMDAR encephalitis}

Marienke A.A.M. de Bruijn, Femke K. Aarsen, Marielle P. van Oosterhout, et al.

Neurology 2018;90;e1997-e2005 Published Online before print April 27, 2018

DOI 10.1212/WNL.0000000000005605

This information is current as of April 27, 2018

Neurology ${ }^{\circledR}$ is the official journal of the American Academy of Neurology. Published continuously since 1951, it is now a weekly with 48 issues per year. Copyright Copyright (C) 2018 The Author(s). Published by Wolters Kluwer Health, Inc. on behalf of the American Academy of Neurology.. All rights reserved. Print ISSN: 0028-3878. Online ISSN: 1526-632X.

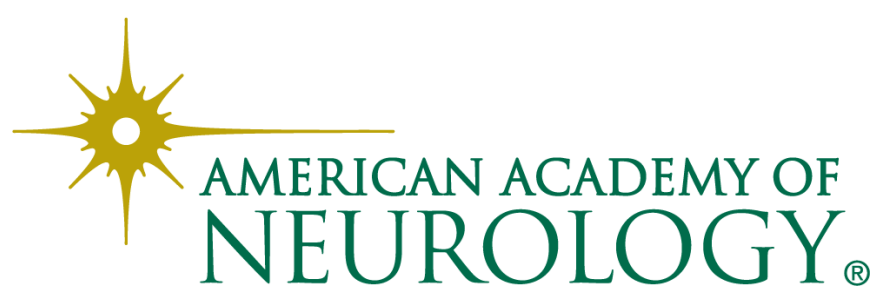




\section{Updated Information \& Services}

\section{Supplementary Material}

\section{References}

Citations

Subspecialty Collections

Permissions \& Licensing

\section{Reprints}

including high resolution figures, can be found at: http://n.neurology.org/content/90/22/e1997.full

Supplementary material can be found at: http://n.neurology.org/content/suppl/2018/05/29/WNL.0000000000005 605.DC1

http://n.neurology.org/content/suppl/2019/12/24/WNL.0000000000005 605.DC2

This article cites 26 articles, 2 of which you can access for free at: http://n.neurology.org/content/90/22/e1997.full\#ref-list-1

This article has been cited by 1 HighWire-hosted articles: http://n.neurology.org/content/90/22/e1997.full\#\#otherarticles

This article, along with others on similar topics, appears in the following collection(s):

\section{All Pediatric}

http://n.neurology.org/cgi/collection/all_pediatric

Autoimmune diseases

http://n.neurology.org/cgi/collection/autoimmune_diseases

Encephalitis

http://n.neurology.org/cgi/collection/encephalitis

Neuropsychological assessment

http://n.neurology.org/cgi/collection/neuropsychological_assessment

Paraneoplastic syndrome

http://n.neurology.org/cgi/collection/paraneoplastic_syndrome

Information about reproducing this article in parts (figures,tables) or in its entirety can be found online at:

http://www.neurology.org/about/about_the_journal\#permissions

Information about ordering reprints can be found online:

http://n.neurology.org/subscribers/advertise

Neurology ${ }^{\circledR}$ is the official journal of the American Academy of Neurology. Published continuously since 1951, it is now a weekly with 48 issues per year. Copyright Copyright ( 2018 The Author(s). Published by Wolters Kluwer Health, Inc. on behalf of the American Academy of Neurology.. All rights reserved. Print ISSN: 0028-3878. Online ISSN: 1526-632X.

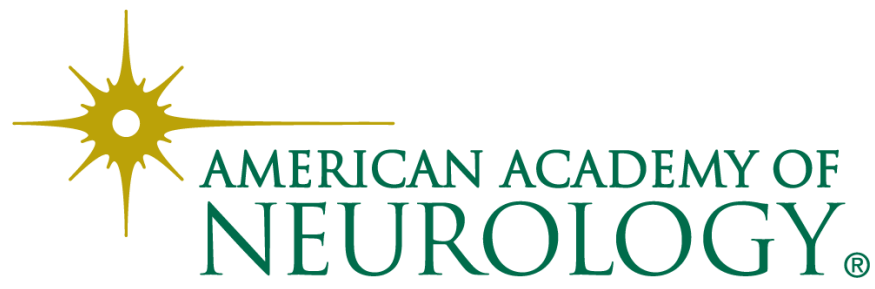

